\title{
Specific and Nonspecific Plasticity of the Primary Auditory Cortex Elicited by Thalamic Auditory Neurons
}

\author{
Xiaofeng Ma and Nobuo Suga \\ Department of Biology, Washington University, St. Louis, Missouri 63130
}

The ventral and medial divisions of the medial geniculate body (MGBv and MGBm) respectively are the lemniscal and nonlemniscal thalamic auditory nuclei. Lemniscal neurons are narrowly frequency tuned and provide highly specific frequency information to the primary auditory cortex (AI), whereas nonlemniscal neurons are broadly frequency tuned and project widely to auditory cortical areas including AI. The MGBv and MGBm are presumably different not only in auditory signal processing, but also in eliciting cortical plastic changes. We electrically stimulated MGBv or MGBm neurons and found the following: (1) electric stimulation of narrowly frequencytuned MGBv neurons evoked the shift of the frequency-tuning curves of AI neurons toward the tuning curves of the stimulated MGBv neurons. This shift was the same as that in the central nucleus of the inferior colliculus and AI elicited by focal electric stimulation of AI or auditory fear conditioning. The widths of the tuning curves of the AI neurons stayed the same or slightly increased. (2) Electric stimulation of broad frequency-tuned MGBm neurons augmented the auditory responses of AI neurons and broadened their frequencytuning curves which did not shift. These cortical changes evoked by MGBv or MGBm neurons slowly disappeared over 45-60 min after the onset of the electric stimulation. Our findings indicate that lemniscal and nonlemniscal nuclei are indeed different in eliciting cortical plastic changes: the MGBv evokes tone-specific plasticity in AI for adjusting auditory signal processing in the frequency domain, whereas the MGBm evokes nonspecific plasticity in AI for increasing the sensitivity of cortical neurons.

\section{Introduction}

The medial geniculate body (MGB) that relays auditory signals from the inferior colliculus (IC) to the auditory cortex (AC) consists of the ventral division (MGBv) of the lemniscal pathway and the medial (MGBm) and dorsal (MGBd) divisions and the posterior intralaminar nucleus (PIN) of the nonlemniscal pathway (Imig and Morel, 1983). The lemniscal pathway is tonotopically organized (Aitkin and Webster, 1972; Clarey et al., 1992), whereas the nonlemniscal pathway is not tonotopically organized and is multisensory (Calford and Webster, 1981; Calford, 1983; Winer and Morest, 1983; Imig and Morel, 1988). Lemniscal neurons are narrowly tuned to a specific single frequency and respond to repetitive tonal stimuli in fidelity. In contrast, nonlemniscal neurons are broadly frequency tuned and rapidly adapt to repetitive identical stimuli (Aitkin and Webster, 1972; Phillips and Irvine, 1979). Lemniscal neurons project to the middle layers of the primary auditory cortex (AI), whereas nonlemniscal neurons broadly project to the superficial layers of the auditory cortical areas including AI (Jones, 1985; Winer, 1992). Different from lemniscal thalamic neurons, nonlemniscal thalamic neurons project to the limbic system such as the amygdala, insular-temporal lobe, and striatum (Shinonaga et al., 1994; Deschênes et al., 1998; Doron and Ledoux, 2000). These anatomical and physiological differences between the lemniscal and nonlemniscal neurons indicate

Received Jan. 12, 2009; revised March 2, 2009; accepted March 18, 2009

Our work has been supported by a research grant from The National Institute on Deafness and Other Communicative Disorders (DC-00175).

Correspondence should be addressed to Xiaofeng Ma, Department of Biology, Washington University, One Brookings Drive, St. Louis, M0 63130. E-mail: xfma@biology.wustl.edu.

D01:10.1523/JNEUROSCI.0167-09.2009

Copyright $\odot 2009$ Society for Neuroscience $\quad$ 0270-6474/09/294888-09\$15.00/0 that they engage in different aspects of auditory signal processing (Aitkin and Prain, 1974; Calford and Aitkin, 1983; Lennartz and Weinberger, 1992) and also in evoking different types of plastic changes (Suga, 2008).

When auditory fear conditioning with a pair of a conditioning tone stimulus (CS) and an unconditioned electric leg or foot stimulus (US) is delivered to an animal such as the guinea pig (for review, see Weinberger, 1998) or big brown bat (for review, see Suga et al., 2002), AI and subcortical auditory neurons shift their frequency tuning curves or receptive fields. That is, they show a best frequency (BF) shift. When the CS and US are unpaired for pseudoconditioning, however, AI and subcortical neurons show nonspecific augmentation (sensitization). How do the lemniscal and nonlemniscal pathways play a role in evoking these two types of cortical plastic changes which are incompatible to each other. Weinberger (1998) hypothesized that the MGBm/PIN evokes the small cortical BF shift, whereas Gao and Suga hypothesized that the MGBv evokes it. Then Suga (2008) further hypothesized that the MGBv evokes the small cortical BF shift whereas the MGBm evokes a small cortical nonspecific augmentation. One of the critical experiments to test these hypotheses is to examine the effect of electric stimulation of narrowly frequency-tuned MGBv neurons or broadly frequency-tuned MGBm neurons on AI neurons. Therefore, we studied changes in AI neurons elicited by electric stimulation of MGBv and MGBm neurons, and found that the MGBv neurons evoked cortical BF shifts, but MGBm neurons did not. Instead, they evoked nonspecific augmentation.

\section{Materials and Methods}

Materials, surgery, acoustic stimulation, electric stimulation, recording of action potentials, and data acquisition and processing were basically 
A

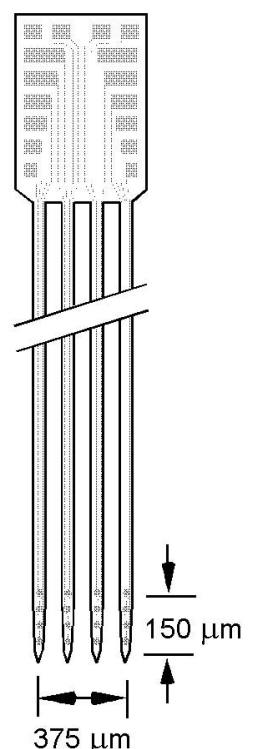

B

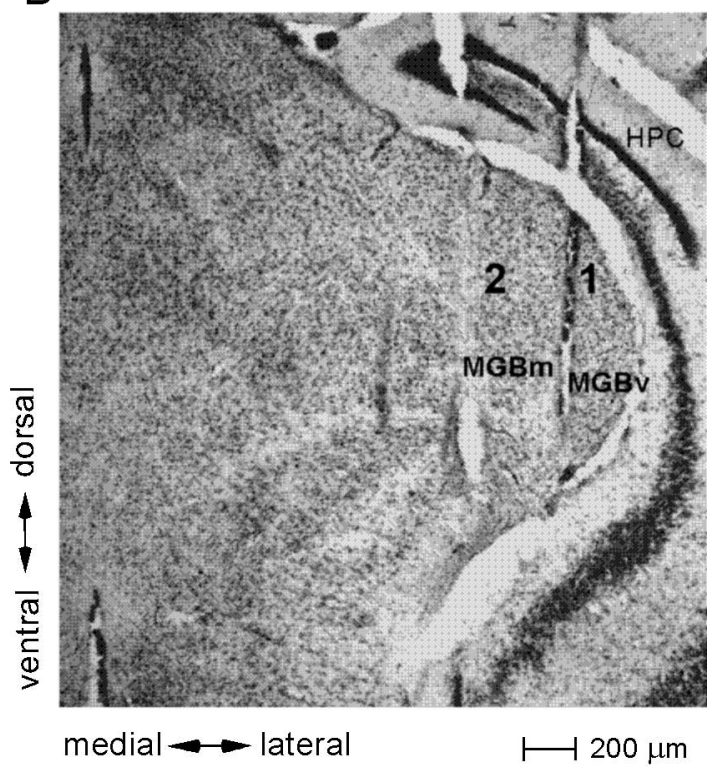

Figure 1. Electrode assembly and recorded/stimulated sites. $A$, An electrode assembly used for recording of action potentials and electric stimulation. $B, A$ coronal section across the medial geniculate body, MGB (Nissl stain, $60 \mu \mathrm{m}$ thick). MGBm, The medial division of the MGB. MGBv, The ventral division of the MGB. 1 and 2, Electrode penetrations across the hippocampus (HPC) and MGBv or MGBm.

the same as those described by Ma and Suga (2001a, 2007), although the site of electric stimulation was the MGB instead of AI. Therefore, only the essential portions of the methods are described below.

Eleven adult big brown bats (Eptesicus fuscus) were used for the current experiments. Under neuroleptanalgesia (Innovar $4.08 \mathrm{mg} / \mathrm{kg}$ b. w.), a 1.5 -cm-long metal post was glued on the dorsal surface of the bat's skull. Physiological experiments were started 3-4 d after the surgery. The awake animal was placed in a polyethylene-foam body mold that was hung with an elastic band at the center of a soundproof room maintained at $\sim 31^{\circ} \mathrm{C}$. The metal post glued on the skull was fixed to a metal rod with set screws to immobilize the animal's head, and the head was adjusted to face directly toward a loudspeaker located $74 \mathrm{~cm}$ away. Holes $(\sim 500 \mu \mathrm{m}$ in diameter) were made in the skull covering the auditory cortex (AC). Through the hole, a multielectrode assembly (Fig. 1A) (NeuroNexus Technologies) was inserted into AI for recording the auditory response (action potentials) of an AI neuron and for studying its frequency tuning. An electrode of the assembly which did not record any auditory responses was used as a reference electrode. Through another hole, another multielectrode assembly was dorsoventrally inserted in a coronal plane into the MGB through the posterior part of $\mathrm{AI}$ and the hippocampus for recording auditory responses (action potentials) of MGB neurons (Fig. $1 \mathrm{~B}$ ), so that we first observed the responses of cortical auditory neurons, regular spontaneous discharges of hippocampal neurons and then the auditory responses of MGB neurons. The electrode which did not record any auditory responses on the same pin was selected as a reference electrode. After recording and studying the auditory responses of MGB neurons, these electrodes were connected to the cathode and anode of the constant current stimulus isolator for electric stimulation. The distance between two electrodes was vertically $50-150 \mu \mathrm{m}$. Because of this vertical short distance, the stimulation was limited in a nucleus. The effect of the electric stimulation on the frequency tuning of the AI neuron was then studied. The protocol for this research was approved by the Animal Studies Committee of Washington University in St. Louis.

Acoustic stimulation. For the measurement of the frequency-threshold (tuning) or -response curve and the best frequency (BF) of a MGB and an AI neuron, a $4.0 \mathrm{~ms}$ tone burst, including a $0.5 \mathrm{~ms}$ rise-decay time, was delivered to the animal from a leaf tweeter (Panasonic, model EAS-10 $\mathrm{TH}-800$ ) at a rate of 5/s. Its frequency and amplitude were varied manually or computer-controlled (TDT System 3, Tucker-Davis Technolo- gies). Before the experiments, the sound amplitude was calibrated with a Brüel \& Kjæel 1/4 inch calibrated microphone (model 4135) placed at the location of the bat's head and was expressed in decibels in sound pressure level (dB SPL) referencing $20 \mathrm{~Pa}$ root-mean-square.

The frequency-tuning curve of a MGB or an AI neuron was first audiovisually measured. Then, the tone burst was randomly changed in amplitude in $5 \mathrm{~dB}$ steps from 0 to $100 \mathrm{~dB}$ SPL and in frequency in $0.5 \mathrm{kHz}$ steps between -5.0 and $5.0 \mathrm{kHz}$ of the $\mathrm{BF}$ if the tuning curve was narrow, but in $1.0 \mathrm{kHz}$ steps between -10 and $10 \mathrm{kHz}$ of the $\mathrm{BF}$ if it was broad, by TDT System 3 (Tucker-Davis Technologies). This frequency-amplitude scan was repeated five times to obtain five responses at each frequency-amplitude combination. It took 7 min to complete the five scans. A frequencytuning curve of a neuron was obtained from its responses to the frequency-amplitude scans. The frequency-response curve of the neuron was obtained by counting the number of action potentials at $30 \mathrm{~dB}$ above the minimum threshold of the neuron as a function of frequency.

Action potentials of AI and MGB neurons. AI neurons were recorded at depths between 300 and $700 \mu \mathrm{m}$ from the cortical surface, whereas MGB neurons were recorded at depths between 2700 and $3300 \mu \mathrm{m}$ from the cortical surface. We used an amplitude-window discriminator software (Tucker-Davis Technologies) to select action potentials of a single neuron. At the beginning of data acquisition, the waveform of an action potential was stored and displayed on the monitor screen. The data acquisition was continued as long as other action potentials obtained during data acquisition matched with this action potential (i.e., template).

Electric stimulation. An electric stimulator (Grass, S88) and a constant current isolator (WPI, A360) were used for focal electric stimulation of the MGB. A $6.2 \mathrm{~ms}$ train of four monophasic electric pulses (100 nA constant current, $0.2 \mathrm{~ms}$ duration, $2.0 \mathrm{~ms}$ interval) was delivered to MGB neurons at a rate of 10/s for $30 \mathrm{~min}$ through two electrodes of the electrode assembly as described above. The bat showed no behavioral response at all to such weak electric stimulation. At the end of the last neurophysiological experiment on an animal, a 10-s-long, $500 \mathrm{nA}$ monophasic electric current was applied to the stimulation electrodes to make a lesion at the stimulated site (Fig. $1 B$ ).

Data acquisition and processing. Data obtained before and after the focal electric stimulation of the MGB were stored on a computer hard drive and were used for off-line data processing that included plotting frequency-tuning and -response curves and measuring the BF, MT, and Q-value at $30 \mathrm{~dB}$ above the minimum threshold of a given neuron. The paired $t$ test was used to test the difference between the responses obtained before and after the electric stimulation.

The frequency-threshold curves of the thalamic neurons could be easily classified into either sharply tuned or multipeaked broadly tuned. However, they should be defined with the quality factors of the curves. So, we calculated a Q-30 dB value (a BF divided by the threshold curve width at $30 \mathrm{~dB}$ above the minimum threshold of a given neuron) instead of a Q-10 dB value, because the Q-10 dB value in a multipeaked tuning curve was large and did not appropriately represent the sharpness of the curve. For each frequency-threshold curve, we defined the broadly tuned neuron when its Q-30 dB was $<6.0$ and the sharply tuned neuron when its Q-30 dB was larger than 9.0. Neurons with a Q-30 dB between 6.0 and 9.0 were classified as intermediately tuned.

\section{Results}

The electrode assembly (Fig. $1 A$ ) was placed 1.3-2.3 mm lateral to the midline and was inserted dorsoventrally into the MGB 
through the posterior portion of $\mathrm{AI}$ and the hippocampus (Fig. $1 B$ ). Auditory responses of MGB neurons were recorded below hippocampal neurons which showed regular spontaneous discharges.

The BFs of the cortical neurons studied ranged from 19 to $56 \mathrm{kHz}(33.1 \pm 6.35$ $\mathrm{kHz}$ ), and the BFs of the MGB neurons studied range from 15 to $55 \mathrm{kHz}(31.4 \pm$ $6.7 \mathrm{kHz})$.

Figure 2 displays the receptive fields of two thalamic ( $A 1$ and $B 1)$ and two cortical (A2-A4 and B2-B4) neurons based upon the responses to the frequency-amplitude scans repeated five times. The thalamic neuron in $A 1$ was sharply tuned to 32.0 $\mathrm{kHz}$, whereas that in $B 1$ was broadly tuned to multiple frequencies. Its BF was 25.0 and/or $28.0 \mathrm{kHz}$. On the other hand, the cortical neurons both were sharply tuned to 29.0 (A2) or 26.0 (B2) $\mathrm{kHz}$. When the sharply tuned thalamic neuron $(A 1)$ was electrically stimulated, the frequencytuning curve (or receptive field) of the cortical neuron sharply tuned to $29.0 \mathrm{kHz}$ (A2) shifted its frequency-tuning curve toward that of the stimulated neuron, so that the BF of this cortical neuron shifted from $29.0 \mathrm{kHz}$ to $30.0 \mathrm{kHz}$. The width of the tuning curve of the cortical neuron slightly widened (A3), so its Q-30 dB changed from 10.6 to 9.7. The shifted BF returned to the control $\mathrm{BF} \sim 60 \mathrm{~min}$ after the onset of the electric stimulation (A4). The BF shift or tuning shift was generally based on a decrease in response (inhibition) at the $\mathrm{BF}$ of the cortical neuron in the control condition and an increase in response (facilitation) at the BF of the stimulated thalamic neuron. Such a BF shift is also elicited by auditory fear conditioning, and has been known as a "tone-specific plastic change" (for review, see Suga and Ma, 2003).

When the broadly tuned thalamic neuron (Fig. 2 B1) was electrically stimulated, the responses of the cortical neuron sharply tuned to $26.0 \mathrm{kHz}$ (B2) were augmented to tone bursts from 18 to $37 \mathrm{kHz}$, so its receptive field was broadened, changing its Q-30 dB from 13.5 to 5.4 (B3). The BF of the cortical neuron did not shift. This augmentation almost disappeared $60 \mathrm{~min}$ after the onset of the electric stimulation (B4). Such augmentation of the responses of cortical neurons occurred over many frequencies even for electric stimulation of the thalamic neurons whose BF was 26 $\mathrm{kHz}$ different from the cortical neurons. Therefore, the augmentation was nonspecific to the BF of the stimulated thalamic neurons. That is, the cortical changes evoked by the broadly tuned thalamic neurons were "nonspecific plastic changes."

The cortical BF shift and nonspecific augmentation evoked by thalamic stimulation are further documented with the frequency-threshold (tuning) and -response curves obtained from cortical neurons different from those shown in Figure 2. In Figure $3 A 1$, the frequency-threshold curve of a thalamic neuron was sharply tuned to $31.0 \mathrm{kHz}$. When this neuron was electrically stimulated, the BF of the sharply tuned cortical neuron $(A 2$, open
B
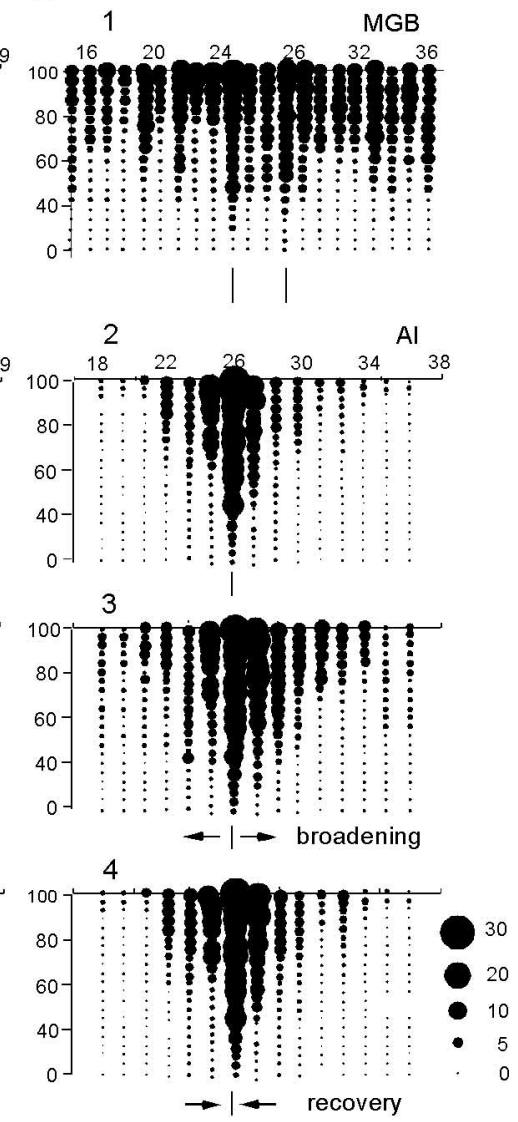

Frequency $(\mathrm{kHz})$

Figure 2. Changes in the receptive fields of two neurons in the primary auditory cortex (Al) evoked by electric stimulation of sharply tuned or broadly tuned neurons in the medial geniculate body (MGB).A1,B1, The receptive fields of the sharply tuned and sharply tuned or broadly tuned. $\boldsymbol{A 4 , B 4}$, Recovery of the receptive fields. Each black dot represents the number of action potentials discharged by the single neuron to five identical stimuli. See the scale at the lower right.

circles) shifted from $33.0 \mathrm{kHz}$ to $32.0 \mathrm{kHz}$ (A2, filled circles). That is, the BF shifted toward the BF of the stimulated thalamic neuron. The shape of the curve changed little. The shifted BF reverted to the control BF 60 min after the onset of the electric stimulation ( $A 2$, dashed line). In Figure 3, B1 and B2, respectively, show the frequency-response curves of these thalamic and cortical neurons obtained at $30 \mathrm{~dB}$ above their minimum thresholds. The frequency-response curves of these neurons were sharply tuned to 31.0 and $33.0 \mathrm{kHz}$, respectively. Electric stimulation of the thalamic neuron evoked a $1.0 \mathrm{kHz}$ shift of the BF of the cortical neuron toward the BF of the stimulated thalamic neuron. The width of the curve changed little (B2, filled circles). The change disappeared $\sim 60$ min after the onset of the electric stimulation (B2, dashed line). From these frequency-threshold and -response curves, it was clear that the sharply tuned thalamic neuron evoked the BF shift of the cortical neuron with little change in the shape of its tuning curves.

Figure 4, $A 1$ and $B 1$, shows the broad frequency-threshold and -response curves of a thalamic neuron, respectively. Its BF was $25.0 \mathrm{kHz}$. Electric stimulation of this neuron evoked nonspecific augmentation of a cortical neuron tuned to $28.5 \mathrm{kHz}$ (Fig. $4 A 2, B 2$, open circles). That is, the frequency-threshold and 


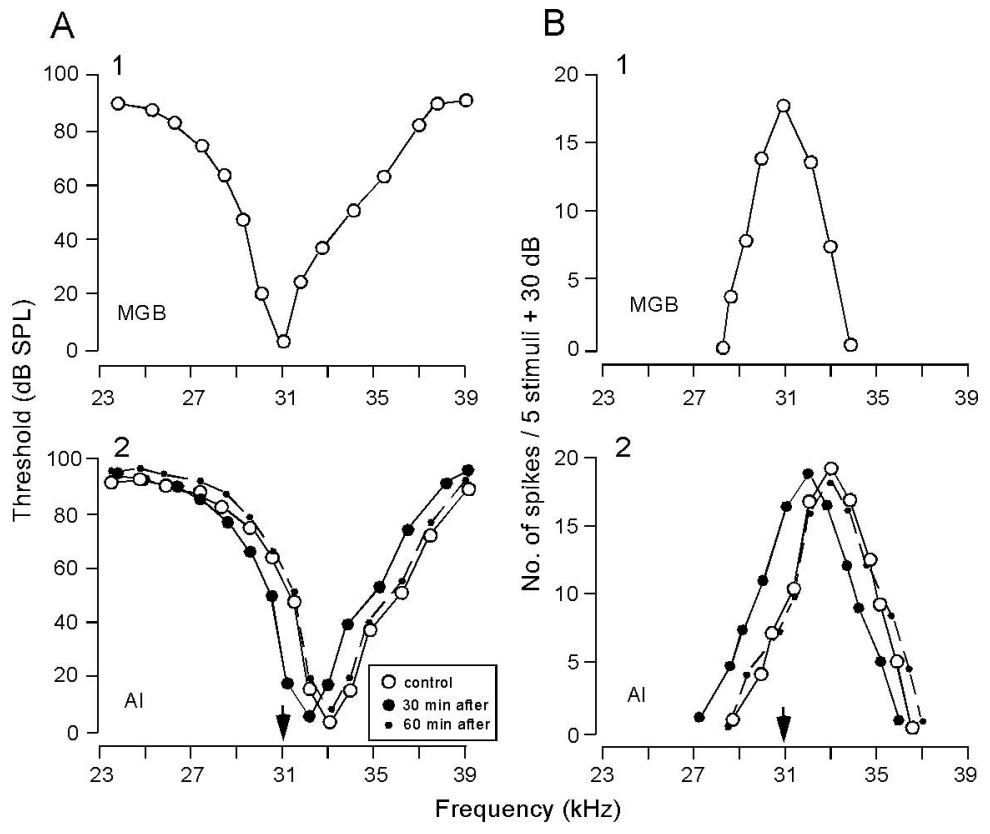

Figure 3. Changes in the frequency-threshold $(\boldsymbol{A})$ and frequency-response $(\boldsymbol{B})$ curves of an Al neuron evoked by electric stimulation of a sharply tuned MGB neuron. Electric stimulation of the MGB neuron $(\boldsymbol{A 1}, \boldsymbol{B 1})$ evoked a shift of the frequencythreshold ( $\boldsymbol{A} \mathbf{2}$ ) and frequency-response (B2) curves of the Al neuron. The open and filled circles represent the curves in the control condition and $30 \mathrm{~min}$ after the onset of the electric stimulation, respectively. The dashed lines represent the curves obtained 60 min after the onset of the electric stimulation. The frequency-response curves were measured at $30 \mathrm{~dB}$ above the minimum threshold of the given neuron.

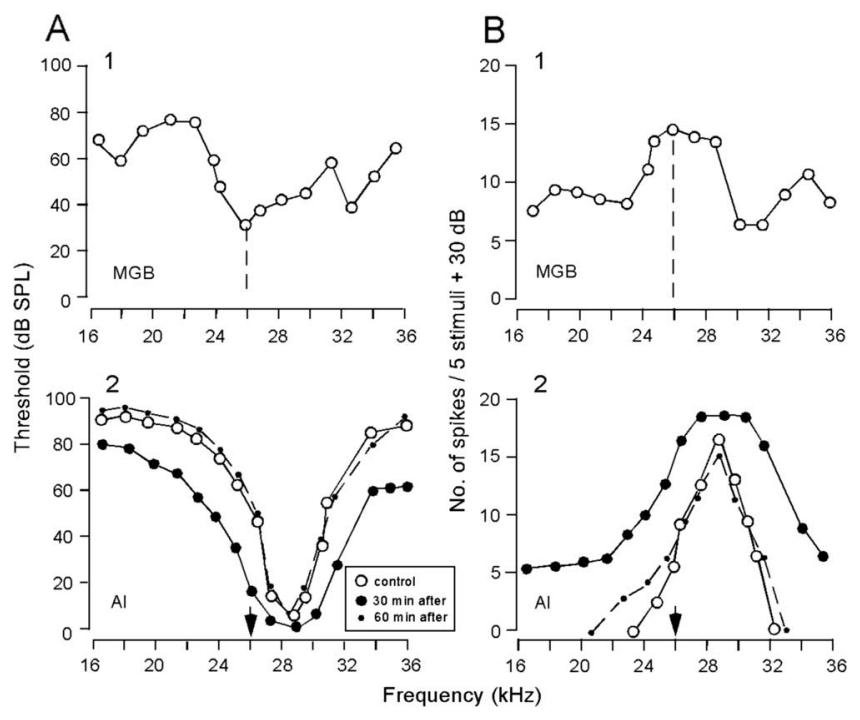

Figure 4. Changes in the frequency-threshold $(\boldsymbol{A})$ or frequency-response $(\boldsymbol{B})$ curves of an $\mathrm{Al}$ neuron evoked by electric stimulation of a broadly tuned MGB neuron. The electric stimulation of the MGB neuron $(\boldsymbol{A 1}, \boldsymbol{B} 1)$ evoked broadening of the frequency-threshold ( $\boldsymbol{A} \mathbf{2})$ and frequencyresponse (B2) curves of the Al neuron. See Figure 3 legend for the symbols.

-response curves of the cortical neuron became broader ( $A 2$ and $B 2$, filled circles). A percentage increase in response was small at the BF but large at frequencies away from the BF. In the control condition, this cortical neuron did not respond to tone bursts $<22 \mathrm{kHz}$ and $>32 \mathrm{kHz}$ at $30 \mathrm{~dB}$ SPL, but responded to those $<22$ $\mathrm{kHz}$ and $>32 \mathrm{kHz}$. The BF did not shift. The broadened curves reverted to the control condition $\sim 60 \mathrm{~min}$ after the onset of the electric stimulation ( $A 2$ and $B 2$, dashed lines). From the changes in these curves, it was clear that, different from the sharply tuned thalamic neuron, the broadly tuned thalamic neuron evoked nonspecific augmentation of the cortical neuron.

In Figure 5, $A$ and $C$, shows the distributions of Q-30 dB values of the 45 stimulated thalamic and 66 recorded cortical neurons. Among the thalamic neurons, 15 had a Q-30 dB smaller than 6.0. Their Q-30 dB values ranged between 2.0 and 6.0 $(3.2 \pm 1.28)$. They were broadly tuned and multipeaked. Their BFs (center frequencies of multipeaked broad threshold curves) ranged from 19 to $55 \mathrm{kHz}(\mathrm{m} \pm$ SD: $33 \pm 3.8 \mathrm{kHz}$ ). Twenty-six thalamic neurons studied had a Q-30 dB larger than 9.0. Their $\mathrm{Q}-30 \mathrm{~dB}$ values ranged between 9.5 and $14(12 \pm 1.5)$. They were sharply tuned to a single frequency. The BFs of these thalamic neurons ranged from 15 to $55 \mathrm{kHz}(\mathrm{m} \pm \mathrm{SD}: 30 \pm 3.9 \mathrm{kHz})$. The mean $\mathrm{BF}$ was not different between the 15 broadly tuned and 26 sharply tuned neurons. Four thalamic neurons had a Q-30 $\mathrm{dB}$ value between 6.0 and 9.0 and are classified as intermediately tuned. The intermediately tuned neurons could be classified as the sharply tuned ones. As described later, however, they showed large facilitation and no BF shifts for the MGB electric stimulation, different from the sharply tuned neurons. When broadly tuned and sharply tuned neurons were simultaneously recorded with an electrode assembly, they were always at the electrodes located medially and laterally, respectively.

These three types of thalamic neurons were differently distributed in the coronal plane across the MGB (Fig. $5 B$ ). That is, the broadly tuned neurons were located $1.3-1.7 \mathrm{~mm}$ lateral from the midline (open circles), whereas the sharply tuned ones were located 1.7-2.3 mm lateral from it (filled circles). The intermediately tuned neurons (Fig. 5, x symbols) were located 1.7-1.9 mm lateral from it. All these neurons were located at depths between 2.7 and $3.3 \mathrm{~mm}$ from the surface of the posterior portion of AI. In terms of the properties of the frequency tuning and anatomical locations, the broadly tuned and sharply tuned neurons may hereafter be called MGBm and MGBv neurons, respectively. In the following, we focus on the effect of electric stimulation of MGBm or MGBv neurons on the frequency tuning of single cortical auditory neurons. As described above, there was no difference in BF between these two groups of thalamic neurons studied, so the difference between these groups in Q-30 dB was not due to any difference in BF.

The BFs of 66 cortical neurons studied ranged from $14 \mathrm{kHz}$ to $45 \mathrm{kHz}(\mathrm{m} \pm \mathrm{SD}: 31 \pm 1.4 \mathrm{kHz})$, and their $\mathrm{Q}-30 \mathrm{~dB}$ values were unimodally distributed from 6.2 to $13(11.5 \pm 1.1)$. According to the above definition, 29 neurons out of the 66 were sharply tuned and the remaining 37 neurons were intermediately tuned (Fig. 5C). Electric stimulation of the thalamic neurons changed the distribution of the Q-30 dB values of the cortical neurons: 43 neurons were intermediately tuned, 17 neurons were sharply tuned, and the remaining six neurons were broadly tuned (Fig. 5B). The increase in the number of the intermediately tuned neurons was mostly due to the stimulation of the MGBm and intermediately tuned neurons. The 
creation of six broadly tuned neurons was all due to the stimulation of the MGBm neurons.

Changes in the Q-30 dB values of the cortical neurons elicited by electric stimulation of the thalamic neurons are plotted in Figure $6 \mathrm{~A}$ as a function of the Q-30 dB values of the thalamic neurons. The cortical neurons were first grouped into BFmatched (filled triangles) and -unmatched neurons. Then, the BF-unmatched neurons were further divided into two groups: BF-shifted (filled circles) and -unshifted (open circles). Electric stimulation of the $\mathrm{MGBv}$ neurons with a $\mathrm{Q}-30 \mathrm{~dB}$ larger than 9.0 evoked a BF shift in 18 cortical neurons (filled circles) and no BF shift in another 18 cortical neurons (open circles). The electric stimulation slightly broadened the cortical frequency-threshold curves, so that the decrease in a Q-30 dB was $3.3 \pm$ $4.5 \%$ for the BF-shifted neurons and $2.8 \pm$ $3.9 \%$ for the BF-unshifted ones. The difference between these two percentage changes was insignificant $(p<0.05)$. On the other hand, electric stimulation of the MGBm neurons with a Q-30 $\mathrm{dB}$ value $<6.0$ and of the MGB neurons with a Q-30 $\mathrm{dB}$ between 6.0 and 9.0 broadened 17 cortical frequencythreshold curves without BF shifts (open circles). An average decrease in a Q-30 dB was $21 \pm 11.1 \%$. Eight BF-matched cortical neurons showed neither BF shifts nor broadening of tuning curves, but two of them showed sharpening of their tuning curves (filled triangles).

$\mathrm{BF}$ shifts depend on the difference in BF between the recorded and stimulated neurons. In the big brown bat, they were not observed when the difference is larger than $10 \mathrm{kHz}$ (Ma and Suga, 2001a). Therefore, all the data shown in Figure $6 A$ were replotted in Figure $6 B$, where the ordinate represents $B F$ differences instead of changes in Q-30 dB values. In Figure $6 B$, two horizontal dashed lines indicate the $10 \mathrm{kHz}$ BF difference between the recorded cortical and stimulated thalamic neurons. For the BF differences $<10 \mathrm{kHz}$ and for the stimulated MGBv neurons, 17 BF-unmatched cortical neurons showed a BF shift and 9 BFunmatched cortical neurons showed no BF shift. Therefore, we may conclude that sharply tuned MGBv neurons evoked the BF shift in AI, whereas the broadly tuned MGBm neurons evoked nonspecific augmentation in AI.

Changes in response magnitude of the cortical neurons evoked by electric stimulation of the thalamic neurons were also examined at the BFs of the cortical neurons in the control condition as the function of Q-30 dB values of the thalamic neurons (Fig. 6C). Electric stimulation of the $17 \mathrm{MGBm}$ and four intermediately tuned MGB neurons increased the responses of the 17 cortical neurons except for one (Fig. 6C, open circles). The amount of the augmentation at the $\mathrm{BF}$ and $30 \mathrm{~dB}$ above the minimum threshold ranged between $0.0 \%$ and $30 \%(15.1 \pm 7.63 \%)$. On the other hand, electric stimulation of MGBv neurons reduced the responses of 15 out of the 18 BF-unmatched and -shifted cortical neurons by $10 \pm 22 \%(7.1 \pm 7.03 \%)$, increased the response of one out of the 18 by $10 \%$, and did not change that of the remaining two (Fig. $6 \mathrm{C}$, filled circles). Electric stimulation of the MGBv neurons increased or decreased the responses of the
BF-unmatched and -unshifted cortical neurons (Fig. 6C, open circles). The mean change was $0.8 \pm 6.09 \%$ (open circles). The BF-matched cortical neurons increased their responses by $\sim 0$ $19 \%(7.0 \pm 5.8 \%)$ when the MGBv neurons (filled triangles) were electrically stimulated. The cortical changes evoked by electric stimulation of the intermediately tuned neurons were different from those evoked by the stimulation of the MGBv neurons: the change in $\mathrm{Q}-30 \mathrm{~dB}$ was $-13.0 \pm 0.01 \%$ for the former, but $-4.0 \pm 0.08 \%$ for the latter $(p=0.02)$ and the change in response magnitude was $18.0 \pm 5.70 \%$ for the former, but $4.1 \pm$ $7.20 \%$ for the latter $(p=0.001)$.

\section{Discussion}

Our current data and the models proposed for the role of MGBv and MGBm in evoking cortical plasticity

It has been repeatedly reported that MGBv neurons are sharply tuned whereas MGBm neurons are broadly tuned and often multipeaked (Aitkin, 1973; Calford, 1983; Bordi and LeDoux, 1994a,b). However, some MGBm neurons are relatively narrowly tuned (Edeline and Weinberger, 1992). Focal electric stimulation of the MGBv evokes BF shifts in AI in the mouse (Jafari et al., 2007) and big brown bat (our current paper). Focal electric stimulation of the central nucleus of the inferior colliculus evokes collicular BF shifts through MGBv, AI, and the corticocollicular projection (Zhang and Suga, 2005). The MGBv shows BF shifts for auditory fear conditioning (Edeline and Weinberger, 1991) and electric stimulation of AI (Zhang et al., 1997; Zhang and Suga, 2000). Therefore, the MGBv undoubtedly is involved in evoking the cortical BF shift.

MGBm neurons of the guinea pig show a BF shift for conditioning (Edeline and Weinberger, 1992). Does this mean that they evoke the cortical BF shift? It had not been examined whether electric stimulation of the MGBm of the guinea pig evoked the cortical BF shift. In our current experiments, we demonstrate that electric stimulation of MGBm neurons 

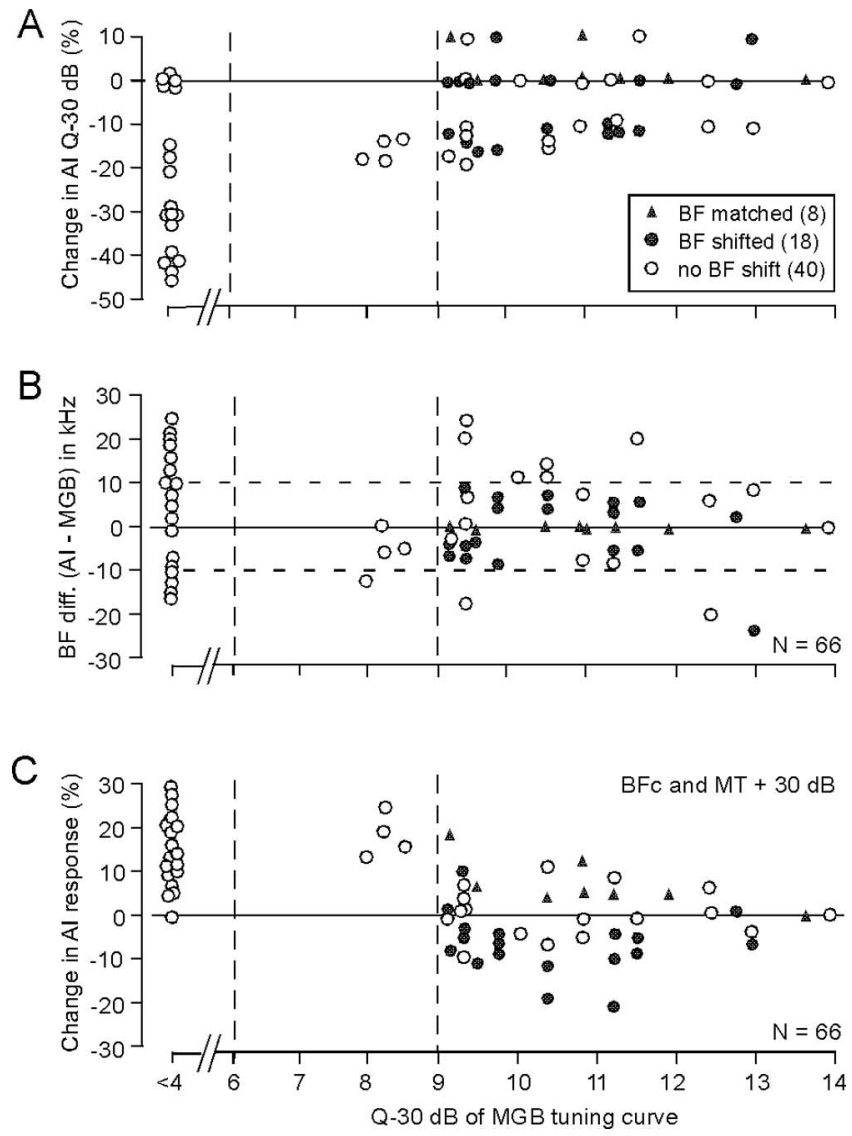

Figure 6. Changes in the sharpness (Q-30 dB) of the frequency-threshold curves of $\mathrm{Al}$ neurons as a function of the sharpness of stimulated MGB neurons $(\boldsymbol{A})$, the distribution of $B F-s h i f t e d$ and BF-unshifted AI neurons in the coordinates of the BF differences between the recorded $A$ and stimulated MGB neurons and the sharpness of the stimulated MGB neurons $(\boldsymbol{B})$, and changes in the response magnitude of Al neurons $(\boldsymbol{C})$ at their $B F$ and 30 $\mathrm{dB}$ above their minimum thresholds in the control condition as a function of the sharpness of the stimulated MGB neurons. Filled triangles, BF-matched AI neurons. Filled circles, BF-unmatched and -shifted Al neurons. Open circles, BF-unmatched and -unshifted AI neurons.

evokes the nonspecific augmentation, not the BF shift, of AI neurons.

Our current data are directly related to the circuit models proposed for the explanation of cortical tone-specific plasticity, i.e., BF shifts. Weinberger $(1998,2004)$ hypothesized that MGBm/PIN evokes the cortical BF shifts only after CS-US association occurs in the MGBm/PIN. In contrast, Gao and Suga (1998) hypothesized that the MGBm/PIN is not involved in evoking the cortical BF shift. Then Suga (2008) further hypothesized that the MGBv and MGBm/PIN are respectively involved in evoking the cortical BF shift (Fig. 7) and nonspecific augmentation (sensitization). The Gao-Suga and Suga models are thus fundamentally different from the Weinberger model. It is clear that our current data support the Gao-Suga and Suga models. In addition, there have been many neurophysiological findings indicating that the large long-term cortical BF shift can be evoked without the activation of the MGBm/PIN by both CS and US (Gao and Suga, 1998; Kilgard and Merzenich, 1998; Weinberger and Bakin, 1998; Yan and Suga, 1998; Ma and Suga, 2001, 2003; Yan and Zhang, 2005; Zhang and Suga, 2005; Jafari et al., 2007; Puckett et al., 2007; Wu and Yan, 2007).

All these three models recognize that ACh released into AI
A

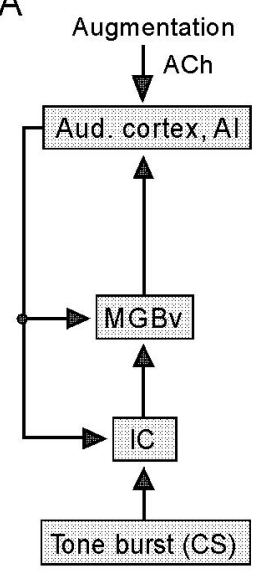

B

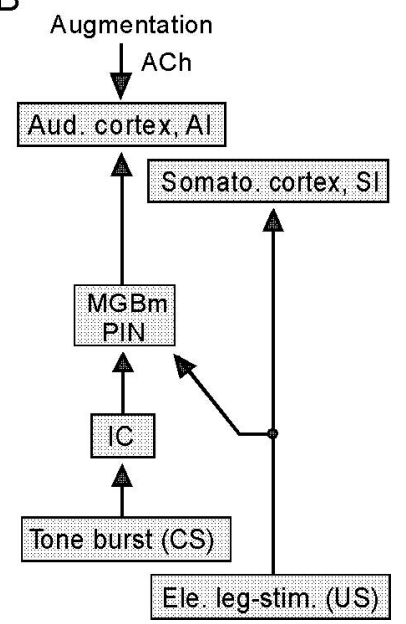

Figure 7. Two models explaining the BF shifts evoked by auditory fear conditioning. $\boldsymbol{A}$, Gao-Suga model: the MGBv is involved in evoking the cortical tone-specific plasticity, i.e., BF shifts. $\boldsymbol{B}$, Weinberger model: the MGBm/PIN is involved in evoking the cortical BF shift only after CS-US association occurs in the MGBm/PIN. In both models, ACh augments the BF shift. ACh, Acetylcholine; Al, primary auditory cortex; CS, conditioning stimulus; IC, inferior colliculus; MGBm and MGBv, the medial and ventral divisions of the medial geniculate body; PIN, posterior intralaminar nucleus; SI, primary somatosensory cortex; TRN, thalamic reticular nucleus; US, unconditioned stimulus.

from the nucleus basalis (NB) augments the small cortical BF shift and changes it into the large long-term BF shift. However, how the NB is activated is different between the Weinberger and Gao-Suga models. In the Weinberger model, the MGBm/PIN sends the CS-US "associated" signal to not only AI but also to the amygdala, which in turn activates the NB. On the other hand the Gao-Suga model states that AC and the somatosensory cortices respectively send CS and US signals to the amygdala, which in turn sends the CS-US associated signal to the NB.

\section{Whether the neural mechanisms found in the big brown bat} are shared with nonbat species

In general, the auditory system of animals is specialized for detecting, processing, and localizing species-specific sounds and sounds produced by prey and predators. Therefore, the auditory system of bats must be specialized for processing biosonar information. This is a common understanding even though one may not know the data indicating it. The auditory system of the mustached bat, Pteronotus parnellii, is one of the best examples of such specialization. Its central auditory system contains two highly specialized subsystems for processing echo delays or Doppler shifts (for review, see Suga, 1990). In the auditory system of the big brown bat, however, it is not clear whether subsystems exist that are specialized for echolocation. There must be differences in the neurophysiological properties of the auditory system between the big brown bat and nonbat species. The differences may be quantitative rather than qualitative.

Compared with nonbat auditory neurons, bat auditory neurons generally respond to higher frequency sounds, respond best to shorter acoustic signals, and respond well to sounds delivered at high repetition rates. However, it is not clear whether the bat data are fundamentally different from the nonbat data because there is no sufficient data for such a comparison. On the other hand, it is easy to enumerate identical or similar data obtained from bat and nonbat species. Therefore, in relation to our current 
research, we enumerate the following facts indicating that the auditory system of the big brown bat shares the identical or similar neurophysiological and morphological properties with that of nonbat species.

(1) As in cats (Imig and Morel, 1883) and rats (Clerici and Coleman, 1990; Clerici et al., 1990), the auditory thalamus consists of multiple nuclei (for review, see Wenstrup and Grose, 1995; our current observation).

(2) As in cats (Aitkin, 1973; Calford, 1983), MGBv and MGBm neurons show sharp and broad frequency-tuning curves, respectively (our current findings).

3) As in cats (Katsuki et al., 1959; Calford et al., 1983), frequency-tuning curves tend to be broader in AI than in the $\mathrm{MGBv}$ (our current findings).

(4) As in the house mouse (Jafari et al., 2007), electric stimulation of MGBv neurons evokes cortical "centripetal" BF shifts, i.e., shifts toward the BF of the stimulated neurons (our current findings).

(5) As in cats and rodents, its AI has a high-to-low frequency axis along the anteroposterior axis of AI (Jen et al., 1989; Dear et al., 1993).

(6) As in the Mongolian gerbil (Sakai and Suga, 2002), focal electric stimulation of AI evokes the center-surround reorganization of the tonotopic-map of AI (Ma and Suga, 2004).

(7) As in the guinea pig (for review, see Weinberger, 1998), auditory fear conditioning elicits cortical and subcortical centripetal BF shifts, i.e., shifts toward the frequency of a conditioned tonal stimulus (Gao and Suga, 2000; Ji et al., 2001).

(8) As in the guinea pig (for review, see Weinberger, 1998) and house mouse (Yan and Ehret, 2002; Yan and Zhang, 2005; Jafari et al., 2007), the cortical and subcortical BF shifts are evoked by focal electric stimulation of the ICc (Zhang and Suga, 2005) or AI (Ma and Suga, 2001a, 2003) or conditioning (Gao and Suga, 2000; Ji et al., 2001) and are augmented by electric stimulation of the nucleus basalis (Ma and Suga, 2003) or by ACh applied to AI (Ji et al., 2001, 2005; Ma and Suga, 2005).

(9) As in the guinea pig (for review, see Weinberger, 1998), the cortical BF shift evoked by the conditioning slowly develops after the conditioning and is long-lasting (Gao and Suga, 2000), and this long-lasting BF shift is due to ACh released into the cortex by the NB (Ji et al., 2001, 2005; Ma and Suga, 2005).

(10) As in the guinea pig (Bakin et al., 1992), pseudoconditioning (unpaired CS and US) elicits nonspecific augmentation (sensitization) in the central auditory system (Ji and Suga, 2008).

(11) The plastic changes in the highly specialized auditory subsystems of the mustached bat are based on the basically identical neural mechanism shared with the auditory systems of many species of mammals including the big brown bat [for review, see Suga and Ma (2003) and Suga (2008)], and with the visual (Tsumoto et al., 1978; Murphy et al., 1999) and somatosensory (Malmierca and Nuñez, 1998; Canedo and Aguilar, 2000) systems.

(12) Duration-tuned neurons are found not only in several species of bats (Pinheiro et al., 1991; Casseday et al., 1994; Galazyuk and Feng, 1997; Ma and Suga, 2001b), but also in cats (He et al., 1997), chinchillas (Chen, 1998), mice (Brand et al., 2000), and frogs (Potter, 1965; Feng et al., 1990; Gooler and Feng, 1992).

(13) FM-sensitive neurons are found not only in several species of bats (Suga, 1965; Fuzessery, 1994), but also in the gerbil (Wetzel et al., 1998), rat (Poon et al., 1991), cat (Glaser, 1971), and monkey (Allon et al., 1981).
(14) Since bats are unique in echolocation, one may be unaware that they use many different types of communication calls, at least 33 types in the mustached bat (Kanwal et al., 1994). In contrast, there is usually only one type of species-specific echolocation call/species. The bats must process these complex calls, as primates do. In the mustached bat, it has been demonstrated that the neurons in the subsystems highly specialized for processing biosonar information respond to its communication calls (Ohlemiller et al., 1996; Kanwal et al., 1999). The bat's auditory system must have the "common" auditory mechanism for processing communication calls. In addition it has the specialized mechanism for processing biosonar signals derived from this common auditory mechanism. The data presented in our current paper are not particularly related to echolocation and indicate a general principle for modulation of the sensory cortex by thalamic sensory neurons.

\section{References}

Aitkin LM (1973) Medial geniculate body of the cat: responses to tonal stimuli of neurons in medial division. J Neurophysiol 36:275-283.

Aitkin LM, Prain SM (1974) Medial geniculate body: unit responses in the awake cat. J Neurophysiol 37:512-521.

Aitkin LM, Webster WR (1972) Medial geniculate body of the cat: organization and responses to tonal stimuli of neurons in ventral division. J Neurophysiol 35:365-380.

Allon N, Yeshurun Y, Wollberg Z (1981) Responses of single cells in the medial geniculate body of awake squirrel monkeys. Exp Brain Res 41:222-232.

Bakin JS, Lepan B, Weinberger NM (1992) Sensitization induced receptive field plasticity in the auditory cortex is independent of CS-modality. Brain Res 577:226-235.

Bordi F, LeDoux JE (1994a) Response properties of single units in areas of rat auditory thalamus that project to the amygdala. I. Acoustic discharge patterns and frequency receptive fields. Exp Brain Res 98:261-274.

Bordi F, LeDoux JE (1994b) Response properties of single units in areas of rat auditory thalamus that project to the amygdala. II. Cells receiving convergent auditory and somatosensory inputs and cells antidromically activated by amygdala stimulation. Exp Brain Res 98:275-286.

Brand A, Urban R, Grothe B (2000) Duration tuning in the mouse auditory midbrain. J Neurophysiol 84:1790-1799.

Calford MB (1983) The parcellation of the medial geniculate body of the cat defined by the auditory response properties of single units. J Neurosci 3:2350-2364.

Calford MB, Aitkin LM (1983) Ascending projections to the medial geniculate body of the cat: evidence for multiple, parallel auditory pathways through thalamus. J Neurosci 3:2365-2380.

Calford MB, Webster WR (1981) Auditory representation within principal division of cat medial geniculate body: an electrophysiology study. J Neurophysiol 45:1013-1028.

Calford MB, Webster WR, Semple MM (1983) Measurement of frequency selectivity of single neurons in the central auditory pathway. Hear Res 11:395-401.

Canedo A, Aguilar J (2000) Spatial and cortical influences exerted on cuneothalamic and thalamocortical neurons of the cat. Eur J Neurosci 12:2515-2533.

Casseday JH, Ehrlich D, Covey E (1994) Neural tuning for sound duration: role of inhibitory mechanisms in the inferior colliculus. Science 264:847-850.

Chen GD (1998) Effects of stimulus duration on responses of neurons in the chinchilla inferior colliculus. Hear Res 122:142-150.

Clarey JC, Barone P, Imig TJ (1992) Physiology of thalamus and cortex. In: The mammalian auditory pathway: neurophysiology (Popper AN, Fay RR, ed), pp 232-334. New York: Springer.

Clerici WJ, Coleman JR (1990) Anatomy of the rat medial geniculate body: I. Cytoarchitecture, myeloarchitecture, and neocortical connectivity. J Comp Neurol 297:14-31.

Clerici WJ, McDonald AJ, Thompson R, Coleman JR (1990) Anatomy of the rat medial geniculate body: II. Dendritic morphology. J Comp Neurol 297:32-54. 
Dear SP, Fritz J, Haresign T, Ferragamo M, Simmons JA (1993) Tonotopic and functional organization in the auditory cortex of the big brown bat, Eptesicus fuscus. J Neurophysiol 70:1988-2009.

Deschênes M, Veinante P, Zhang ZW (1998) The organization of corticothalamic projections: reciprocity versus parity. Brain Res Brain Res Rev 28:286-308.

Doron NN, Ledoux JE (2000) Cells in the posterior thalamus project to both amygdala and temporal cortex: a quantitative retrograde double-labeling study in the rat. J Comp Neurol 425:257-274.

Edeline JM, Weinberger NM (1991) Subcortical adaptive filtering in the auditory system: associative receptive field plasticity in the dorsal medial geniculate body. Behav Neurosci 105:154-175.

Edeline JM, Weinberger NM (1992) Associative retuning in the thalamic source of input to the amygdala and auditory cortex: receptive field plasticity in the medial division of the medial geniculate body. Behav Neurosci 106:81-105.

Feng AS, Hall JC, Gooler DM (1990) Neural basis of sound pattern recognition in anurans. Prog Neurobiol 34:313-329.

Fuzessery ZM (1994) Response selectivity for multiple dimensions of frequency sweeps in the pallid bat inferior colliculus. J Neurophysiol 72:1061-1079.

Galazyuk AV, Feng AS (1997) Encoding of sound duration by neurons in the auditory cortex of the little brown bat, Myotis lucifugus. J Comp Physiol [A] 180:301-311.

Gao E, Suga N (1998) Experience-dependent corticofugal adjustment of midbrain frequency map in bat auditory system. Proc Natl Acad Sci U S A 95:12663-12670.

Gao E, Suga N (2000) Experience-dependent plasticity in the auditory cortex and the inferior colliculus of bats: role of the corticofugal system. Proc Natl Acad Sci U S A 97:8081-8086.

Glaser EM (1971) Cortical responses of awake cat to narrow-band FM noise stimuli. J Acoust Soc Am 50:490-501.

Gooler DM, Feng AS (1992) Temporal coding in the frog auditory midbrain: the influence of duration and rise-fall time on the processing of complex amplitude-modulated stimuli. J Neurophysiol 67:1-22.

He J, Hashikawa T, Ojima H, Kinouchi Y (1997) Temporal integration and duration tuning in the dorsal zone of cat auditory cortex. J Neurosci 17:2615-2625.

Imig TJ, Morel A (1983) Organization of the thalamocortical auditory system in the cat. Annu Rev Neurosci 6:95-120.

Imig T, Morel A (1988) Organization of the cat's auditory thalamus. In: Auditory function: neurobiological bases of hearing (Edelman G, Gall WE, Cowan WM, ed), pp 457-485. New York: Wiley.

Jafari MR, Zhang Y, Yan J (2007) Multiparametric changes in the receptive field of cortical auditory neurons induced by thalamic activation in the mouse. Cereb Cortex 17:71-80.

Jen PH, Sun XD, Lin PJ (1989) Frequency and space representation in the primary auditory cortex of the frequency modulating bat Eptesicus fuscus. J Comp Physiol [A] 165:1-14.

Ji W, Suga N (2008) Tone-specific and nonspecific plasticity of the auditory cortex elicited by pseudoconditioning: role of acetylcholine receptors and the somatosensory cortex. J Neurophysiol 100:1384-1396.

Ji W, Gao E, Suga N (2001) Effects of acetylcholine and atropine on plasticity of central auditory neurons caused by conditioning in bats. J Neurophysiol 86:211-225.

Ji W, Suga N, Gao E (2005) Effects of agonists and antagonists of NMDA and ACh receptors on plasticity of bat auditory system elicited by fear conditioning. J Neurophysiol 94:1199-1211.

Jones E (1985) The thalamus. New York: Plenum.

Kanwal JS, Matsumura S, Ohlemiller K, Suga N (1994) Analysis of acoustic elements and syntax in communication sounds emitted by mustached bats. J Acoust Soc Am 96:1229-1254.

Kanwal JS, Fitzpatrick DC, Suga N (1999) Facilitatory and inhibitory frequency tuning of combination-sensitive neurons in the primary auditory cortex of mustached bats. J Neurophysiol 82:2327-2345.

Katsuki Y, Watanabe T, Maruyama N (1959) Activity of auditory neurons in upper levels of brain of cat. J Neurophysiol 22:343-359.

Kilgard MP, Merzenich MM (1998) Cortical map reorganization enabled by nucleus basalis activity. Science 279:1714-1718

Lennartz RC, Weinberger NM (1992) Frequency selectivity is related to temporal processing in parallel thalamocortical auditory pathways. Brain Res 583:81-92.
Ma X, Suga N (2001a) Plasticity of bat's central auditory system evoked by focal electric stimulation of auditory and/or somatosensory cortices. J Neurophysiol 85:1078-1087.

Ma X, Suga N (2001b) Corticofugal modulation of duration-tuned neurons in the midbrain auditory nucleus in bats. Proc Natl Acad Sci U S A 98:14060-14065.

Ma X, Suga N (2003) Augmentation of plasticity of the central auditory system by the basal forebrain and/or somatosensory cortex. J Neurophysiol 89:90-103.

Ma X, Suga N (2004) Lateral inhibition for center-surround reorganization of the frequency map of bat auditory cortex. J Neurophysiol 92:3192-3199.

Ma X, Suga N (2005) Long-term cortical plasticity evoked by electric stimulation and acetylcholine applied to the auditory cortex. Proc Natl Acad Sci U S A 102:9335-9340.

Ma X, Suga N (2007) Multiparametric corticofugal modulation of collicular duration-tuned neurons: modulation in the amplitude domain. J Neurophysiol 97:3722-3730.

Malmierca E, Nuñez A (1998) Corticofugal action on somatosensory response properties of rat nucleus gracilis cells. Brain Res 810:172-180.

Murphy PC, Duckett SG, Sillito AM (1999) Feedback connections to the lateral geniculate nucleus and cortical response properties. Science 286:1552-1554

Ohlemiller KK, Kanwal JS, Suga N (1996) Facilitative responses to speciesspecific calls in cortical FM-FM neurons of the mustached bat. Neuroreport 7:1749-1755.

Phillips DP, Irvine DR (1979) Acoustic input to single neurons in pulvinarposterior complex of cat thalamus. J Neurophysiol 42:123-136.

Pinheiro AD, Wu M, Jen PH (1991) Encoding repetition rate and duration in the inferior colliculus of the big brown bat, Eptesicus fuscus. J Comp Physiol [A] 169:69-85.

Poon PW, Chen X, Hwang JC (1991) Basic determinants for FM responses in the inferior colliculus of rats. Exp Brain Res 83:598-606.

Potter HD (1965) Patterns of acoustically evoked discharges of neurons in the mesencephalon of the bullfrog. J Neurophysiol 28:1155-1184.

Puckett AC, Pandya PK, Moucha R, Dai W, Kilgard MP (2007) Plasticity in the rat posterior auditory field following nucleus basalis stimulation. J Neurophysiol 98:253-265.

Sakai M, Suga N (2002) Centripetal and centrifugal reorganizations of frequency map of auditory cortex in gerbils. Proc Natl Acad Sci U S A 99:7108-7112.

Shinonaga Y, Takada M, Mizuno N (1994) Direct projections from the nonlaminated divisions of the medial geniculate nucleus to the temporal polar cortex and amygdala in the cat. J Comp Neurol 340:405-426.

Suga N (1965) Analysis of frequency-modulated sounds by auditory neurones of echo-locating bats. J Physiol 179:26-53.

Suga N (1990) Biosonar and neural computation in bats. Sci Am 262:60-68.

Suga N (2008) Role of corticofugal feedback in hearing. J Comp Physiol [A] 194:169-183.

Suga N, Ma X (2003) Multiparametric corticofugal modulation and plasticity in the auditory system. Nat Rev Neurosci 4:783-794.

Suga N, Xiao Z, Ma X, Ji W (2002) Plasticity and corticofugal modulation for hearing in adult animals. Neuron 36:9-18.

Tsumoto T, Creutzfeldt OD, Legéndy CR (1978) Functional organization of the corticofugal system from visual cortex to lateral geniculate nucleus in the cat (with an appendix on geniculo-cortical mono-synaptic connections). Exp Brain Res 32:345-364.

Weinberger NM (1998) Physiological memory in primary auditory cortex: characteristics and mechanisms. Neurobiol Learn Mem 70:226-251.

Weinberger NM (2004) Specific long-term memory traces in primary auditory cortex. Nat Rev Neurosci 5:279-290.

Weinberger NM, Bakin JS (1998) Learning-induced physiological memory in adult primary auditory cortex: receptive fields plasticity, model, and mechanisms. Audiol Neurootol 3:145-167.

Wenstrup JJ, Grose CD (1995) Inputs to combination-sensitive neurons in the medial geniculate body of the mustached bat: the missing fundamental. J Neurosci 15:4693-4711.

Wetzel W, Ohl FW, Wagner T, Scheich H (1998) Right auditory cortex lesion in Mongolian gerbils impairs discrimination of rising and falling frequency-modulated tones. Neurosci Lett 252:115-118. 
Winer J (1992) The functional architecture of the medial geniculate body and the primary auditory cortex. In: The mammalian auditory pathway: neuroanatomy (Webster D, Popper AN, Fay RR, eds), pp 222-409. New York: Springer.

Winer JA, Morest DK (1983) The medial division of the medial geniculate body of the cat: implications for thalamic organization. J Neurosci 3:2629-2651.

Wu Y, Yan J (2007) Modulation of the receptive fields of midbrain neurons elicited by thalamic electrical stimulation through corticofugal feedback. J Neurosci 27:10651-10658.

Yan J, Ehret G (2002) Corticofugal modulation of midbrain sound processing in the house mouse. Eur J Neurosci 16:119-128.
Yan J, Zhang Y (2005) Sound-guided shaping of the receptive field in the mouse auditory cortex by basal forebrain activation. Eur J Neurosci 21:563-576

Yan W, Suga N (1998) Corticofugal modulation of the midbrain frequency map in the bat auditory system. Nat Neurosci 1:54-58.

Zhang Y, Suga N (2000) Modulation of responses and frequency tuning of thalamic and collicular neurons by cortical activation in mustached bats. J Neurophysiol 84:325-333.

Zhang Y, Suga N (2005) Corticofugal feedback for collicular plasticity evoked by electric stimulation of the inferior colliculus. J Neurophysiol 94:2676-2682.

Zhang Y, Suga N, Yan J (1997) Corticofugal modulation of frequency processing in bat auditory system. Nature 387:900-903. 